DRAFT VERSION NOVEMBER 4, 2018

Preprint typeset using LTEX style emulateapj v. 03/07/07

\title{
SN 2005AP: A MOST BRILLIANT EXPLOSION
}

\author{
Robert M. Quimby ${ }^{1}$, Greg Aldering ${ }^{2}$, J. Craig Wheeler ${ }^{1}$, Peter HöFlich ${ }^{3}$, Carl W. Akerlof ${ }^{4}$, Eli S. RykofF ${ }^{4}$ \\ Draft version November 4, 2018
}

\begin{abstract}
We present unfiltered photometric observations with ROTSE-III and optical spectroscopic follow-up with the HET and Keck of the most luminous supernova yet identified, SN 2005ap. The spectra taken about 3 days before and 6 days after maximum light show narrow emission lines (likely originating in the dwarf host) and absorption lines at a redshift of $z=0.2832$, which puts the peak unfiltered magnitude at $-22.7 \pm 0.1$ absolute. Broad P-Cygni features corresponding to $\mathrm{H} \alpha, \mathrm{C}$ III, N III, and O III, are further detected with a photospheric velocity of $\sim 20,000 \mathrm{~km} \mathrm{~s}^{-1}$. Unlike other highly luminous supernovae such as $2006 \mathrm{gy}$ and $2006 \mathrm{tf}$ that show slow photometric evolution, the light curve of SN 2005ap indicates a 1-3 week rise to peak followed by a relatively rapid decay. The spectra also lack the distinct emission peaks from moderately broadened (FWHM $\sim$ $2,000 \mathrm{~km} \mathrm{~s}^{-1}$ ) Balmer lines seen in SN 2006gy and SN 2006tf. We briefly discuss the origin of the extraordinary luminosity from a strong interaction as may be expected from a pair instability eruption or a GRB-like engine encased in a $\mathrm{H} / \mathrm{He}$ envelope.
\end{abstract}

Subject headings: Supernovae, SN 2005ap

\section{INTRODUCTION}

Luminous supernovae ( $\mathrm{SNe})$ are most commonly associated with the Type Ia class, which are thought to involve explosions of white dwarf stars; however, the brightest $\mathrm{SNe}$ (those with absolute magnitudes $M<-20$ ) are all associated with the deaths of stars that begin their lives with main sequence masses $M_{\mathrm{ms}}>7-8 M_{\odot}$. The current record holder, SN 2006gy, is thought to be an explosion of a supermassive $\operatorname{star}\left(M_{\mathrm{ms}} \sim 100-150 M_{\odot}\right)$ and it may represent the first detection of a supernova triggered by pair instability (Smith et al. 2006). The remaining bright $\mathrm{SNe}$ are attributed to corecollapse induced explosions (CCSNe).

$\mathrm{CCSNe}$ are classified as Type Ib/c if they lack strong hydrogen lines in their spectra and Type II otherwise (Filippenko 1997). The latter class has been partitioned into three groups: (1) Type IIn show narrow emission lines in their spectra, (2) Type II-L have linearly declining light curves, and (3) Type II$\mathrm{P}$ are "normal" hydrogen rich events exhibiting a slow photometric evolution phase (i.e. a plateau). SN 1993J is sometimes classified as a "Type IIb" as its spectral characteristics evolved from Type II-like to resemble a Type Ib, which lack $\mathrm{H}$ but show He lines (unlike the Type Ic class). CCSNe may span a continuum with the different spectral types explained in terms of decreasing envelope masses (II-IIb-Ib-Ic).

Long Duration gamma-ray bursts (GRBs) are occasionally observed to exhibit SN features. These appear as bumps in the optical afterglow light curves (Bloom et al. 1999; Lazzati et al.2001), or more revealingly as broad spectral features resembling SNe Ic that emerge in the spectra of afterglows 1-2 weeks after the burst (Stanek et al.2003). Given the connection of GRBs to SNe Ic and the continuum of CCSNe spectral types, it is natural to consider the observational signature of a GRB engine erupting within an envelope of some mass (e.g. MacFadyen et al. 2001). Such material could slow

\footnotetext{
${ }^{1}$ Department of Astronomy, University of Texas, Austin, TX 78712, USA

2 Physics Division, LBNL, 1 Cyclotron Road, Berkeley, CA 94720, USA

3 Department of Physics, Florida State University, Tallahassee, FL 32312 USA

${ }^{4}$ University of Michigan, 2477 Randall Laboratory, 450 Church Street, Ann Arbor, MI, 48104, USA
}

the ultra-relativistic flow and thus mask the gamma-ray beacon announcing their creation, unlike their stripped progenitor cousins. Young et al. (2005) have explored this possibility and constructed models that can explain the light curves of the bright subclass within the Type II-L group.

In this letter we report the discovery of SN 2005ap, a transient optical source that peaked at about -22.7 magnitude. We present the photometry in $\$ 2$ the spectral observations and modeling in $\$ 3$ and we offer discussion and conclusions in $\$ 4$ SN 2005ap is the brightest supernova ever identified and may shed light on energy production mechanisms in cosmic explosions.

\section{PHOTOMETRY}

SN 2005ap was discovered on unfiltered optical images taken with the $0.45 \mathrm{~m}$ ROTSE-IIIb (Robotic Optical Transient Search Experiment) telescope (Akerlof et al. 2003), which is located at the McDonald observatory in west Texas. The transient was found on 2005 March 3 (UT dates are used throughout this letter) in images taken in the course of the Texas Supernova Search (TSS; Quimby 2006). SN 2005ap was identified in a field centered on the Coma Galaxy Cluster after removal of static sources via a modified version of the image subtraction code developed by the Supernova Cosmology Project (Perlmutter et al. 1999). SN 2005ap is located at $\alpha=13^{h} 01^{m} 14^{\mathrm{s}} .83, \delta=+27^{\circ} 43^{\prime} 32^{\prime \prime} \cdot 3$ (J2000), which is 6.2" projected from SDSS J130115.12+274327.5 (galaxy A in Fig. 1); however, Adami et al. (2006) have cataloged a source $0.6^{\prime \prime} \pm 0.3^{\prime \prime}$ west of the transient with $B=24.6 \pm 0.2$, $V=23.9 \pm 0.2$, and $R=23.5 \pm 0.3$, and we identify this as the host of SN 2005ap. SN 2005ap reached an unfiltered magnitude of $18.13 \pm 0.08$ (calibrated against the USNO-B1.0 R2 values ${ }^{5}$ ) on March 10.28 and was observed at a similar brightness the following two nights (see Fig. 2). The transient was detected over a 40 day period, and it has since remained undetected over two years of observations by the ROTSE-III telescopes (170 nights with limiting magnitudes $\left.18<M_{\text {lim }}<19.5\right)$. A $4^{\text {th }}$ order polynomial fit to the detections shows maximum light occurred on 2005 March 10.6.

5 http://www . nofs . navy -mil/data/fchpix/ 


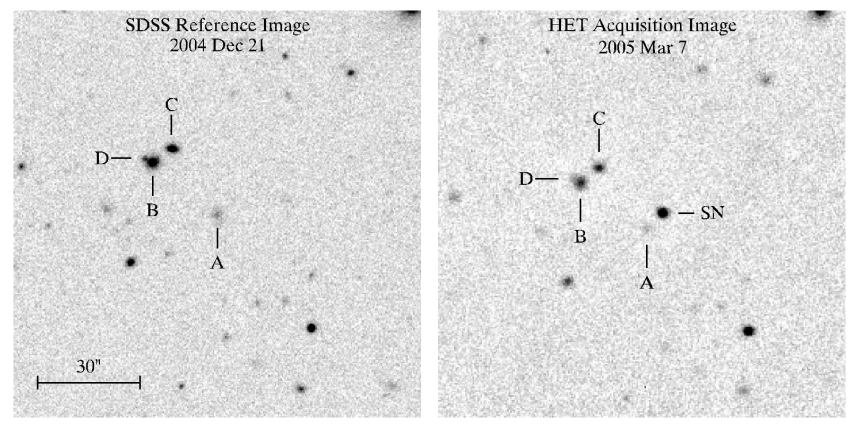

FIG. 1.- SDSS reference image (left) and HET/LRS acquisition image of SN 2005ap (right). The SDSS image is a combination of the $g-, r$-, and $i$-band frames taken 80 days before maximum light. The host is not clearly detected on the combined SDSS frame, but four neighboring galaxies are labeled A-D. The $\mathrm{SN}$ is marked on the HET/LRS image.

SN 2005ap brightened over at least a 7 day period to reach its peak. The shape of the light curve is consistent with supernova templates that take $\sim 3$ weeks to reach maximum light.

\section{SPECTROSCOPY AND SPECTRAL FITTING}

A spectrum of SN 2005ap acquired 2005 March 7 with the Low Resolution Spectrograph (LRS; Hill et al. 1998) on the Hobby-Eberly Telescope (HET) shows a very blue continuum with subtle line features including broad absorption and at least one narrow emission line (Fig. 3). Keck/LRIS (Oke et al. 1995) data obtained 9 days later exhibit similar behavior, and additionally show narrow absorption lines at wavelengths not covered by the HET. We identify the narrow emission lines observed around 6363 and $6425 \AA$ as [O III] $\lambda 4959,5007$, and a narrow emission line around $8422 \AA$ in the Keck spectrum as $\mathrm{H} \alpha$. The narrow absorption doublet seen at $3595 \AA$ corresponds to $\mathrm{Mg}$ II $\lambda 2796,2803$ in this same frame, and sets a firm lower limit for the redshift of SN 2005ap at $z=0.2832$. At this redshift, the peak unfiltered magnitude is $-22.7 \pm 0.1$ absolute $\left(\Omega_{m}=0.265\right.$, $\left.\Omega_{\Lambda}=0.735, H_{0}=71 \mathrm{~km} \mathrm{~s}^{-1} \mathrm{Mpc}^{-1}\right)$. Corrections for minor $\left(A_{R}=0.022^{m}\right)$ Galactic extinction (Schlegel et al. 1998) and absorption within the host are not included.

The alignment of the slit for the HET data includes the southern edge of galaxy B, which is $25.7^{\prime \prime}$ from the SN (Fig. 11). These spectra indicate that the redshift of galaxy $\mathrm{B}$ is consistent with the Mg II absorption doublet at $3525 \AA$ $(z \sim 0.26$; projected separation $\sim 100 \mathrm{kpc})$. The remaining $\mathrm{Mg}$ II absorption system is consistent with the SDSS photometric redshift of galaxy $\mathrm{C}$ in Figure 1, to within the uncertainty (Adelman-McCarthy et al. 2007).

A second observation was conducted with the HET on 2007 February 23, long after the transient had faded below the detection limit of ROTSE-III. The slit was aligned to include the position of SN 2005ap as well as galaxy A. The [O III] $\lambda 4959,5007$ doublet is clearly detected near the location of the transient (Fig. 4). This may indicate that the narrow emission lines are not directly associated with SN 2005ap, but rather are intrinsic to the faint host, which has an absolute magnitude, $M_{R}$, of about -16.8 . Like the host, galaxy $\mathrm{A}$ is also redshifted by $z \sim 0.283$, giving it an absolute magnitude of about $M_{R}=-20.4$ and the pair a projected separation of $28.3 \mathrm{Kpc}$.

We used the parameterized spectral synthesis code, SYNOW (Jeffery \& Branch 1990), to identify broad features in the spectra of SN 2005ap. First we note that if the continuum is a black body, then the steep dependence with wave-

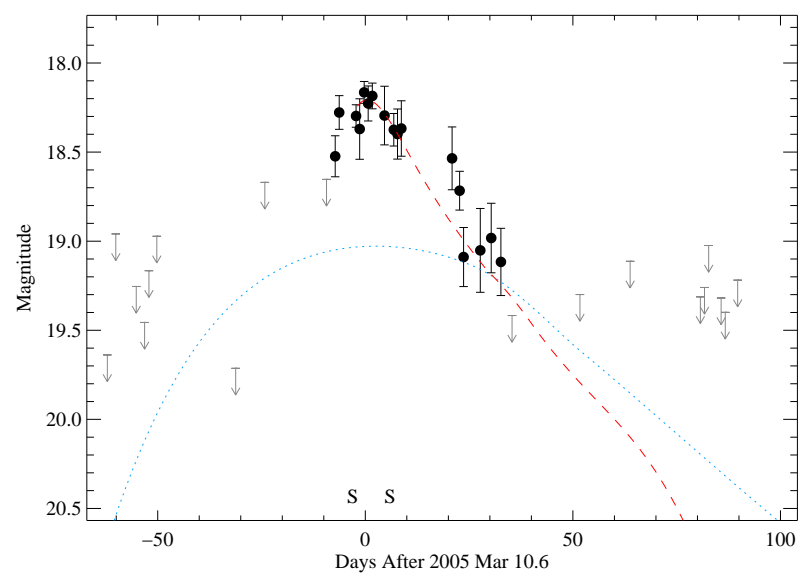

FIG. 2.- ROTSE-III unfiltered light curve of SN 2005ap calibrated against the USNO-B1.0 R2 magnitudes. Detections are indicated by filled circles. Upper limits fainter than 18.5 mag prior to discovery and 19.0 after the last detection are marked with arrows. The red dashed line depicts the $V$-band light curve of a SN II-L (SN 1980K [Barbon et al. 1982; Buta 1982]) dilated in time by $1+z$ and arbitrarily shifted by 4 magnitudes to match the peak. The dotted blue line is the unfiltered light curve of SN 2006gy from Smith et al. (2006), corrected for its 1.68 magnitudes of extinction and phased to match the peak of SN 2005ap, as it would appear at a redshift of $z=0.2832$. The HET and Keck spectral epochs are each marked along the abscissa with an "S."

length implies a 16,000 to $20,000 \mathrm{~K}$ source. Alternatively, the continuum may be a power-law, $f_{\nu} \propto \nu^{\beta}$, with $\beta \sim 0.7$. We paid special attention to the extraction of the blue channel Keck data. The rollover at the blue end cannot signal a black body peak within the allowed temperature range. The spectra are modeled as $20,000 \mathrm{~K}$ and $17,000 \mathrm{~K}$ black body continua for the March 7 and 16 data, respectively, with superposed P-Cygni profiles.

The lack of distinct features in the 4500 to $8000 \AA$ range imposes significant constraints on the cause of the broad absorption features between rest 3500 and $4500 \AA$. We obtain acceptable fits to both spectral epochs from SYNOW using only H I, C III, N III, and O III (colored lines in Fig. 3). He II may also be present, but its strongest feature is blended with $\mathrm{C} \mathrm{III/N} \mathrm{III} \mathrm{and}$ is not strictly required. The presence of such high excitation species is consistent with the high temperatures implied by the blue continuum slope. SYNOW indicates the C III feature at about $2600 \AA$ is temperature dependent, and C III can acceptably fit the rollover at the blue end of the Keck spectrum if the ion temperature is lowered to about $7,500 \mathrm{~K}$ (the apparent inconsistency between the ion and continuum temperatures may arise due to assumptions in the SYNOW code, which neglects non-LTE effects). Between March 7 and March 16 the lines shift redward by 50-60 (note the HET and Keck wavelength solutions agree to $\sim 0.1 \%$ ). This indicates a significant deceleration of the photosphere between the two epochs. The SYNOW fits give a photospheric velocity of $\sim 23,000 \mathrm{~km} \mathrm{~s}^{-1}$ on March 7, decreasing to $\sim 19,000 \mathrm{~km} \mathrm{~s}^{-1}$ on March 16 .

\section{DISCUSSION AND CONCLUSIONS}

We have presented photometric and spectroscopic observations of the Type II supernova 2005ap. Narrow absorption and emission lines from within the dwarf host indicate a redshift of $z=0.2832$. The successful SYNOW fits and the lack of additional narrow absorption systems indicate the SN cannot be far beyond this redshift. Correcting only for distance, the peak unfiltered magnitude was $-22.7 \pm 0.1$ absolute, which corresponds to $\sim 4 \times 10^{44} \mathrm{erg} \mathrm{s}^{-1}$ assuming no bolometric correc- 


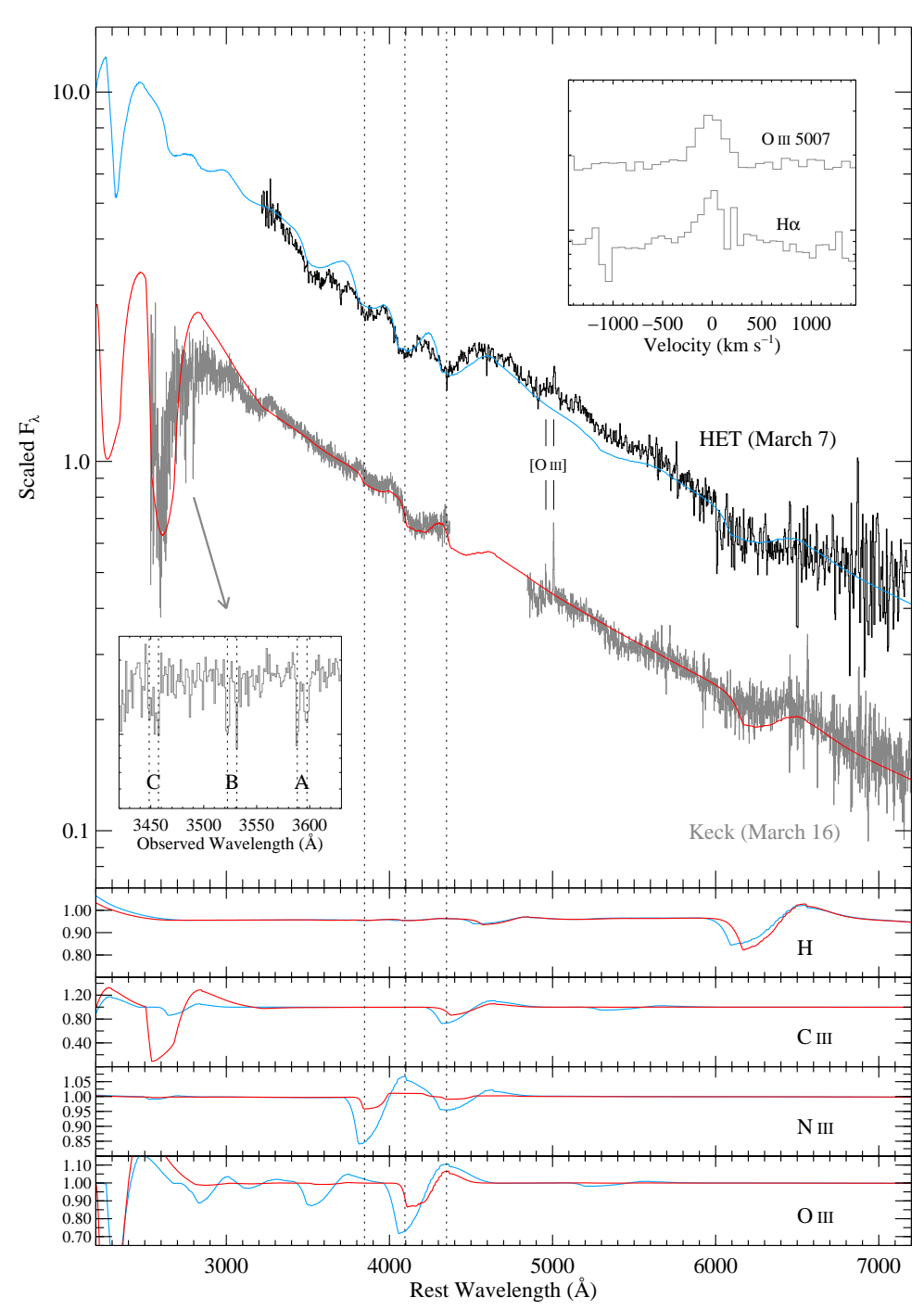

FIG. 3.- HET/LRS spectrum of SN 2005ap taken 3 days before maximum light (black curve) and the Keck/LRIS data from 6 days after maximum light (grey curve). The blue and red curves show spectral fits to the data from the highly parameterized SYNOW code. An unidentified P-Cygni profile is seen around $3200 \AA$. The left inset is a detail from the Keck spectra showing three narrow absorption doublets that we identify as intervening $\mathrm{Mg}$ II $\lambda 2796,2804$ systems. The upper inset shows the narrow $\mathrm{H} \alpha$ and [O III] $\lambda 5007$ from the Keck data plotted in velocity space relative to the $z=0.2832$ restframe. The bottom panels show the individual contributions from the four ions included in the fits.

tion. We consider below the possibility that SN 2005ap was an AGN or a GRB afterglow, but we find it is most consistent with a supernova explosion. We conclude SN 2005ap is the most luminous supernova ever identified, and roughly twice as bright as the previous record holder, SN 2006gy.

Although the peak absolute magnitude of SN 2005ap is comfortably in the range $-21>M>-23$ typical of X-ray selected AGN around this redshift (Anderson et al. 2007), this interpretation is unlikely. First and foremost, this transient occurred in a region of the sky that has been exceptionally well observed across the electromagnetic spectrum for decades; no indication of a possible AGN at this position has previously been uncovered. Specifically, there are no X-ray detections identified in either the ROSAT All-Sky Survey source catalogs (Voges et al. 1999, 2000) or the XMM-Newton Serendip-

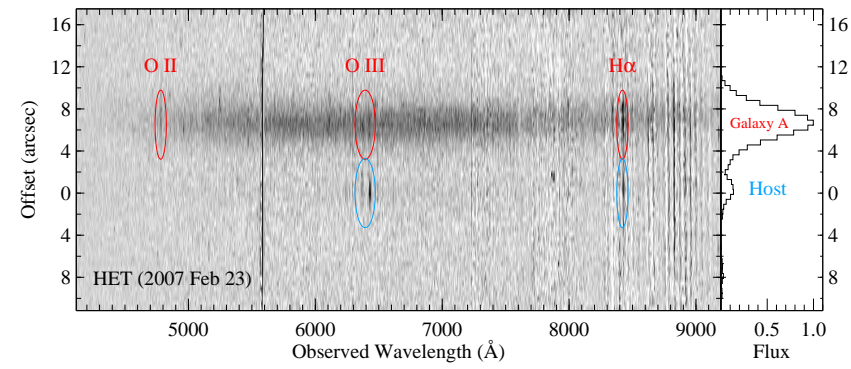

FIG. 4.- HET/LRS spectra taken about two years after SN 2005ap faded below the detection limit of ROTSE-III. The slit was oriented to include the location of the SN as well as galaxy A. The narrow [O III] $\lambda 4959,5007$ doublet redshifted by $z=0.283$ is still clearly present (larger blue circle), suggesting this emission was not associated with SN 2005ap. Galaxy A also exhibits the [O III] doublet as well as [O III] $\lambda 3727$ (red circles). $\mathrm{H} \alpha$ is detected in both galaxies, although it is confused with the imperfectly subtracted night sky lines. In addition to the narrow emission lines, the host is detected as a weak continuum source. The integrated light is plotted in the right panel. Note the host emission is distinct from galaxy A.

itous Source Catalogue ${ }^{6}$, nor is there a cataloged radio source present in either the NRAO VLA Sky Survey (Condon et al. 1998) or the FIRST survey catalog (White et al. 1997). Second, the spectra are quite different from normal AGN, showing no $\mathrm{H} \beta$ or $\mathrm{Mg}$ II emission features. There is some similarity to the featureless spectra of certain blazars, although blazars lack P-Cygni line profiles and often show narrow lines from giant elliptical hosts (Wurtz et al. 1996). Third, the best position for SN 2005ap is offset from its host at the $2 \sigma$ level, and at peak the transient was more than 5 magnitudes brighter than the underlying host light. Finally, no repeat outbursts have been detected at this location in two years of monitoring.

The host galaxy is reminiscent of settings for long duration GRBs (Fruchter et al. 2006); however, the observed population of GRB afterglows typically decline as power-laws ( $f \propto t^{\alpha}$ with $\alpha \sim-1$ ), and the 1-3 week rise and slow fading of SN 2005ap are inconsistent with this trend. An off-axis orphan afterglow is a interesting possibility, although current models predict a rapid power-law decline after maximum light with $\alpha \sim-1.5$ (Nakar \& Piran 2003), which does not appear to match the observations.

Except for its unusually bright peak magnitude, the observations of SN 2005ap are quite consistent with the typical behavior of supernovae. The time scales for the photometric evolution are common among $\mathrm{SNe}$, as is the presence of broad, P-Cygni profiles in the spectra. There have been SNe with continua slopes about as blue as SN 2005ap (e.g. SN 1998S; Fassia et al. 2001), but the existence of C III, N III, and $\mathrm{O}$ III features is unprecedented. The broad $\mathrm{H} \alpha$ P-Cygni profile is of course the defining feature of SNe II. With no narrow emission lines clearly associated with the explosion and lacking an observed photometric plateau phase, SN 2005ap could be classified as a Type II-L. The light curve is similar in shape to the behavior of SNe II-L (Fig. 2) although it is 3-4 mag too bright. The spectra are also roughly similar to the early observations of SN 1979C (Branch et al. 1981), except SN 2005ap shows a much bluer continuum. However, SNe II-L show broad, asymmetric $\mathrm{H} \alpha$ emission with little to no blueshifted absorption, and they also have $\mathrm{H} \beta, \mathrm{H} \gamma$, and $\mathrm{He}$ I/Na I P-Cygni profiles. These characteristics are wanting in the observations of SN 2005ap. While most SNe II-L have narrowly distributed peak magnitudes, a small subsam-

6 http://xcatdb.u-strasbg.fr/xcatdb-corr/ 
ple (including SN 1979C) deviate to brighter values (Gaskell 1992). Young et al. (2005) have suggested these deviants are powered by a GRB engine within a H/He envelope.

The luminosity of normal SNe II is powered by energy deposited by the explosion shock, but this requires that the initial radius of the progenitor be significant compared to the radius of the photosphere in order to minimize adiabatic losses. For an exceptionally bright object like SN 2005ap, this would require an unrealistically large radius. With a photosphere moving at about $\mathrm{v}_{p h} \sim 20,000 \mathrm{~km} \mathrm{~s}^{-1}$ and a time of maximum light of $\mathrm{t}_{\text {max }} \sim 1 \times 10^{6} \mathrm{~s}$, the radius of the photosphere near maximum light is $\mathrm{R}_{p h} \sim 2 \times 10^{15} \mathrm{~cm}$, which is much too large to correspond to a standard red supergiant.

If the luminosity arises in the collision of the ejecta with a surrounding, perhaps dense, shell of circumstellar matter shed by a wind or a process like an LBV mass ejection, then we might see the radiation emitted by a shocked, thermalized, shell. For a shell of mass $\mathrm{M}_{s h}$, radius $\mathrm{R}$, thickness $\Delta \mathrm{R}$, and optical depth, $\tau$, maximum light will occur when the diffusion time, $\mathrm{t}_{\text {diff }} \sim 3 \Delta R \tau / \mathrm{c}$, is comparable to the dynamical time, $\mathrm{t}_{d y n} \sim \Delta \mathrm{R} / \mathrm{v}_{p h}$. This gives the optical depth at maximum light, $\tau_{\max } \sim 1 / 3\left(\mathrm{c} / \mathrm{v}_{p h}\right) \sim 5$ neglecting any differences between the photospheric velocity and the mean ejecta velocity. Since $\mathrm{M}_{s h} \sim 4 \pi \mathrm{R}^{2} \tau_{\max } / \kappa$, the mass of the shell would be about $1.3 \mathrm{M}_{\odot}$, with $\kappa=0.2$ for electron scattering. The peak luminosity would be $\mathrm{L} \sim 1 / 2 \mathrm{M}_{s h} \mathrm{v}_{p h}^{2} / \mathrm{t}_{\max } \sim 2 \times 10^{45} \mathrm{erg} \mathrm{s}^{-1}$, more than directly observed, but roughly consistent with the observations. The model for SNe II-L by Young et al. (2005) is a variation on this theme, but with the optical luminosity produced by non-thermal emission from the afterglow process.

Another possibility is that a separate internal source of energy powers the luminosity. A model driven purely by radioactive decay would require a large asymmetry to prevent the nickel mass $\left(10\right.$ 's of $\left.\mathrm{M}_{\odot}\right)$ from exceeding the ejecta mass (Höflich et al. 1999), which must be modest to account for the rapid rise and decline of the light curve. Whether this could be accomplished with a large nickel mass suggesting a pair formation event, or with a lower nickel mass more commensurate with a "normal" core collapse explosion requires more extended analysis.

Maeda et al. (2007) propose the rapid spin-down of a mag- netar to produce a high intrinsic luminosity and subsequent decline from the second peak of SN 2005bf. It is possible that an appropriate choice of ( $a d$ hoc) parameters could produce the peak we observe in SN 2005ap, but with a smaller mass and larger temporal decay index to account for the brighter, faster peak.

In two years of operation, the Texas Supernova Search has uncovered the most luminous supernova (this work), the second most luminous (SN 2006gy [Smith et al.2006; Ofek et al. 2007]), and a third, highly luminous event (SN 2006tf [Quimby et al. 2007]). The volume monitored over this time (roughly matching the total from all past narrow-field CCD surveys targeting known galaxies), the high completeness of the sample resulting from a tight (nightly) cadence and ability to work in the cores of galaxies, along with prompt high $\mathrm{S} / \mathrm{N}$ spectroscopic follow-up, have been key to finding and fully classifying these events. The lack of SN 2005ap-like events reported in previous surveys may suggest they are intrinsically rare, but the lack of SN 2006gy-like events might be understood if they are preferentially located in the cores of bright galaxies. Our discovery of new classes of bright transients bodes well for more extensive future surveys of the time-variable sky.

We would like to thank the staff of the Hobby-Eberly Telescope and McDonald Observatory for their support. We give specific thanks to V. Riley and B. Roman for their observations with the HET, and to F. Castro, P. Mondol, and M. Sellers for their efforts in screening potential SN candidates. We thank M. Kowalski and D. Schlegel for their assistance with the Keck observations, and D. Branch and J. Parrent for discussions on the SYNOW code. This research is supported, in part, by NASA grant NAG 5-7937 (PH), NSF grants AST0307312 (PH) and AST0406740 (RQ \& JCW), and DOE contract DE-AC02-05CH11231 (GA). The authors wish to recognize and acknowledge the very significant cultural role and reverence that the summit of Mauna Kea has always had within the indigenous Hawaiian community. We are most fortunate to have the opportunity to conduct observations from this mountain.

\section{REFERENCES}

Adami, C., Picat, J. P., Savine, C., et al. 2006, A\&A, 451, 1159

Adelman-McCarthy, J. K., Agueros, M. A., Allam, S. S., et al. 2007, in press Akerlof, C. W., Kehoe, R. L., McKay, T. A., et al. 2003, PASP, 115, 132

Anderson, S. F., Margon, B., Voges, W., et al. 2007, AJ, 133, 313

Barbon, R., Ciatti, F., \& Rosino, L. 1982, A\&A, 116, 35

Bloom, J. S., Kulkarni, S. R., Djorgovski, S. G., et al. 1999, Nature, 401, 453

Branch, D., Falk, S. W., Uomoto, A. K., et al. 1981, ApJ, 244, 780

Buta, R. J. 1982, PASP, 94, 578

Condon, J. J., Cotton, W. D., Greisen, E. W., et al. 1998, AJ, 115, 1693

Fassia, A., Meikle, W. P. S., Chugai, N., et al. 2001, MNRAS, 325, 907

Filippenko, A. V. 1997, ARA\&A, 35, 309

Fruchter, A. S., Levan, A. J., Strolger, L., et al. 2006, Nature, 441, 463

Gaskell, C. M. 1992, ApJ, 389, L17

Hill, G. J., Nicklas, H. E., MacQueen, P. J., et al. 1998, in Proc. SPIE Vol. 3355, p. 375-386, Optical Astronomical Instrumentation, Sandro D’Odorico; Ed., 375-386

Höflich, P., Wheeler, J. C., \& Wang, L. 1999, ApJ, 521, 179

Jeffery, D. J. \& Branch, D. 1990, in Supernovae, Jerusalem Winter School for Theoretical Physics, ed. J. C. Wheeler, T. Piran, \& S. Weinberg, 149-+ Lazzati, D., Covino, S., Ghisellini, G., et al. 2001, A\&A, 378, 996
MacFadyen, A. I., Woosley, S. E., \& Heger, A. 2001, ApJ, 550, 410 Maeda, K., Tanaka, M., Nomoto, K., et al. 2007, ArXiv e-prints, 705 Nakar, E. \& Piran, T. 2003, New Astronomy, 8, 141

Ofek, E. O., Cameron, P. B., Kasliwal, M. M., et al. 2007, ApJ, 659, L13

Oke, J. B., Cohen, J. G., Carr, M., et al. 1995, PASP, 107, 375

Perlmutter, S., Aldering, G., Goldhaber, G., et al. 1999, ApJ, 517, 565

Quimby, R., Castro, F., \& Mondol, P. 2007, IAU Circ., 8790, 2

Quimby, R. M. 2006, PhD thesis, University of Texas, United States - Texas Schlegel, D. J., Finkbeiner, D. P., \& Davis, M. 1998, ApJ, 500, 525

Smith, N., Li, W., Foley, R. J., et al. 2006, ArXiv Astrophysics e-prints

Stanek, K. Z., Matheson, T., Garnavich, P. M., et al. 2003, ApJ, 591, L17

Voges, W., Aschenbach, B., Boller, T., et al. 1999, A\&A, 349, 389

—. 2000, VizieR Online Data Catalog, 9029, 0

White, R. L., Becker, R. H., Helfand, D. J., \& Gregg, M. D. 1997, ApJ, 475, 479

Wurtz, R., Stocke, J. T., \& Yee, H. K. C. 1996, ApJS, 103, 109

Young, T. R., Smith, D., \& Johnson, T. A. 2005, ApJ, 625, L87 